\title{
Symptombelastung, Inanspruchnahme des Gesundheitssystems und Todesumstände von Menschen mit Demenz in der letzten Lebensphase: der Bayerische Demenz Survey (BayDem)
}

\section{Symptom Burden, Health Services Utilization and Places and Causes of Death in People with Dementia at the End of Life: the Bavarian Dementia Survey (BayDem)}

\section{(ㅇ)(1) $\odot \odot$}

\author{
Autoren \\ André Kratzer ${ }^{1}$, Linda Karrer², Nikolas Dietzel ${ }^{2}$, Franziska Wolff², Manuela Hess², Peter Kolominsky-Rabas², \\ Elmar Gräßel ${ }^{1}$
}

Institute

1 Zentrum für Medizinische Versorgungsforschung, Psychiatrische und Psychotherapeutische Klinik, Universitätsklinikum Erlangen, Friedrich-AlexanderUniversität Erlangen-Nürnberg (FAU), Erlangen

2 Interdisziplinäres Zentrum für Health Technology Assessment (HTA) und Public Health (IZPH), FriedrichAlexander-Universität Erlangen-Nürnberg (FAU), Erlangen

\section{Schlüsselwörter}

Demenz, Palliativversorgung, Lebensende, Inanspruchnahme, Symptombelastung, Tod

Key words

Dementia, Palliative care, End of life, Symtom burden, Utilization, Death

\section{Bibliografie}

DOI https://doi.org/10.1055/a-1033-7159

Online-Publikation: 20.12.2019

Gesundheitswesen 2020; 82: 50-58

(c) Georg Thieme Verlag KG Stuttgart - New York

ISSN 0941-3790

\section{Korrespondenzadresse}

André Kratzer, M.Sc. Psych.

Universitätsklinikum Erlangen

Psychiatrische und Psychotherapeutische Klinik

Zentrum für Medizinische Versorgungsforschung

Schwabachanlage 6

91054 Erlangen

andre.kratzer@uk-erlangen.de

\section{ZUSAMMENFASSUNG}

Hintergrund Demenz ist in der Regel eine lebenslimitierende Erkrankung. Dennoch fehlen derzeit evidenzbasierte Leitlinien für eine angemessene Palliativversorgung von Menschen mit Demenz (MmD). Ein Grund hierfür ist der Mangel an belastbaren empirischen Daten zu MmD in der letzten Lebensphase. Ziel dieser Untersuchung ist daher, Symptombelastung, Inanspruchnahme des Gesundheitssystems sowie Todesumstände von MmD in der letzten Lebensphase zu beschreiben.

Methodik Der Bayerische Demenz Survey (BayDem) war eine multizentrische Längsschnittstudie, die in 3 Regionen Bayerns (Dachau, Erlangen, Kronach) durchgeführt wurde. Teilnehmende waren MmD nach ICD-10 sowie deren pflegende Angehörige. Die Verlaufsdaten wurden in standardisierten, persönlichen Interviews vor Ort erhoben. Es erfolgte ein 1:1-Propensity Score Matching zwischen verstorbenen und nicht verstorbenen MmD. Zur statistischen Analyse wurden McNemar-Tests sowie $\mathrm{t}$-Tests für verbundene Stichproben verwendet.

Ergebnisse In der vorliegenden Analyse wurden 58 im Studienzeitraum verstorbene und 58 nicht verstorbene $\mathrm{MmD}$ untersucht $(n=116)$. In den meisten Fällen sind $M m D$ zuhause (36,2\%), im Krankenhaus (25,9\%) oder im Alten-/Pflegeheim $(19,0 \%)$ verstorben, nie im Rahmen einer Palliativversorgung. Todesursachen waren meist Komplikationen des respiratorischen Systems (13,8\%), kardiovaskuläre Komplikationen $(12,1 \%)$ sowie Schlaganfälle (12,1\%). MmD in der letzten Lebensphase wiesen stärker ausgeprägte körperliche Komorbiditäten auf als die übrigen $\mathrm{MmD}$ (Charlson-Index: $\mathrm{M}=2,75$ vs. $M=1,80 ; p=0,030$, Cohen's $d=0,425$ ) und wurden dementsprechend häufiger in ein Krankenhaus eingewiesen (46,6 vs. $12,1 \%, p<0,001, O R=6,250)$ und in einer Notaufnahme $(22,4$ vs. $3,4 \%, p=0,007, O R=6,500$ ) behandelt. Psychische und Verhaltensauffälligkeiten waren stark ausgeprägt (NPI-Wert: $M=31,67$ vs. $M=24,77, p=0,118$, Cohen's $d=0,303$ ). Ambulante Angebote wurden jedoch selten in Anspruch genommen. Schlussfolgerungen Die Ergebnisse unterstreichen die Notwendigkeit, evidenzbasierte Leitlinien für eine angemessene, 
den speziellen Bedürfnissen von MmD in der letzten Lebensphase entsprechende, Palliativversorgung zu entwickeln. Die starke Ausprägung an psychischen und Verhaltensauffälligkeiten sollte dabei ebenso wie die stark ausgeprägten körperlichen Komorbiditäten berücksichtigt werden. Angesichts häufiger Krankenhausaufenthalte sollte zudem ein Fokus auf die Entwicklung fachlicher Empfehlungen für den stationären Bereich (Akutkrankenhaus, Palliativstation) gelegt werden.

\section{ABSTRACT}

Background Dementia is usually a life-limiting disease. However, evidence-based guidelines for palliative care for people with dementia (PwD) are currently lacking. One reason for this is the dearth of reliable empirical data on PwD at the end of life. The aim of this study is to describe the symptom burden, causes of death, places of death and the use of various health services for PwD at the end of life.

Methods The Bavarian Dementia Survey (BayDem) was a multi-center, longitudinal study at 3 different sites in Bavaria, Germany (Dachau, Kronach, Erlangen). Participants were PwD defined by ICD-10 and their informal caregivers. Data were collected in standardized face-to-face interviews in cooperation with local actors. In order to obtain comparable groups, deceased and non-deceased PwD were matched using 1:1 pro- pensity score matching. For the statistical analyses, McNemar tests as well as paired t-tests were used.

Results In this analysis, 58 deceased and 58 non-deceased PwD were studied ( $n=116)$. In most cases, PwD died at home (36.2\%), in hospital (25.9\%) or in a nursing home (19.0\%), but no one in palliative care. The most common causes of death were respiratory (13.8\%) and cardiovascular complications (12.1\%) as well as stroke (12.1\%). PwD at the end of life showed more pronounced physical comorbidities than the other PwD (CharlsonIndex: $\mathrm{M}=2.75$ vs. $\mathrm{M}=1.80 ; \mathrm{p}=0.030$, Cohen's $\mathrm{d}=0.425$ ) and were therefore admitted to hospital ( 46.6 vs. $12.1 \%, \mathrm{p}<0.001$, $\mathrm{OR}=6.250)$ or emergency departments (22.4 vs. $3.4 \%$, $\mathrm{p}=0.007, \mathrm{OR}=6.500$ ) more frequently. Behavioral and psychological symptoms were very pronounced (NPI Score: $M=31.67$ vs. $M=24.77, p=0.118$, Cohen's $d=0.303$ ). Nevertheless, the utilization of outpatient health services was low.

Conclusion The results underline the need to develop evidence-based guidelines to provide palliative care specifically adapted to the needs of PwD at the end of life. In this context, the high incidence of behavioral and psychological symptoms should be taken into account, as should the high incidence of physical comorbidities. Considering the frequent hospital admissions, special attention should also be paid to the development of recommendations for the inpatient sector (acute hospital and palliative care unit).

\section{Einleitung}

Demenz ist mit etwa 47 Mio. Betroffenen weltweit eine der zentralen gesellschaftlichen Herausforderungen unserer Zeit [1]. Allein in Deutschland leben derzeit 1,7 Mio. Menschen mit Demenz (MmD) [2]. Aufgrund der steigenden Lebenserwartung gehen aktuelle Prognosen von einem Anstieg der Prävalenz in Deutschland auf etwa 3 Mio. und international auf rund 131,5 Mio. Menschen im Jahr 2050 aus [1, 2].

Da eine kurative Behandlung von Demenz bisher nicht existiert, kann man Demenz in der Regel als lebenslimitierende Erkrankung betrachten [3]. Die primären unmittelbaren Todesursachen sind in diesem Zusammenhang in den meisten Fällen assoziierte Komplikationen wie eine Kachexie bzw. Dehydrierung, kardiovaskuläre Störungen und Störungen des respiratorischen Systems, insbesondere eine Pneumonie [4].

Aufgrund der hohen Symptomlast von MmD am Lebensende, die neben in der Regel schweren kognitiven Einschränkungen und körperlich-neurologischen Beeinträchtigungen auch psychische und Verhaltensauffälligkeiten umfasst [3, 5], ist eine adäquate Palliativversorgung für diese Zielgruppe zwingend erforderlich [6].

Trotz der mittlerweile zunehmenden Forderungen nach einer adäquaten Palliativversorgung von MmD am Lebensende existieren hierfür bis dato keine evidenzbasierten Empfehlungen bzw. Leitlinien [3, 5-8]. Erste Ratschläge gibt lediglich das White Paper der European Association for Palliative Care [6], welches auf Basis einer Befragung von Experten erstellt wurde. Erklärt werden kann dieser Zustand dadurch, dass sich die Palliativversorgung in Deutschland $[3,7]$, aber auch international $[6,8]$ bisher vorwie- gend auf Menschen mit Tumorerkrankungen fokussierte, wenngleich im Zuge der Erweiterung der WHO Definition von Palliative Care [9] ein Zuwachs an empirischen Studien zur Palliativversorung von bspw. Menschen mit Herzinsuffizienz [10] oder chronisch obstruktiven Lungenerkrankungen [11] zu verzeichnen war. Dennoch mangelt es weiterhin an empirischen Daten zur Versorgungssituation und Symptomlast von MmD in der letzten Lebensphase [3, 5, 8] sowie qualitativ hochwertiger Forschung zu wirksamen Interventionen im Rahmen der Palliativversorgung dieser Zielgruppe [12].

Ziel dieses Artikels ist es deshalb, Symptombelastung, Todesursachen, Sterbeorte sowie die Inanspruchnahme verschiedener Versorgungsleistungen des Gesundheitssystems von MmD in der letzten Lebensphase zu beschreiben und aufzuzeigen, in welchen Aspekten sie sich von anderen MmD unterscheidet.

\section{Methodik}

\section{Studiendesign und Studienpopulation}

Der Bayerische Demenz Survey (BayDem) war eine multizentrische Längsschnittstudie in 3 Regionen in Bayern [13]. Die 3 Regionen Dachau, Erlangen und Kronach stellen verschiedene demografische und sozioökonomische Gebiete mit unterschiedlichen Bevölkerungsentwicklungen dar. Durch Einbeziehung der Städte und Landkreise wurden sowohl urbane als auch ländliche Räume abgebildet. Teilnehmende waren MmD (definiert nach ICD-10, F00-F03 [14]), die von ihren pflegenden Angehörigen zuhause versorgt wurden, 
wie auch die Angehörigen selbst. Die vorliegende Arbeit fokussiert ausschließlich auf die Zielgruppe MmD.

\section{Rekrutierung und Follow-up}

Um den unterschiedlichen Zugangswegen von MmD Rechnung zu tragen, erfolgte die Rekrutierung der Teilnehmenden über vielfältige Einrichtungen (Beratungsstellen, niedergelassene Ärztinnen und Ärzte sowie Therapeutinnen und Therapeuten, medizinische Versorgungszentren, Gedächtnisambulanzen, Pflegedienste, ehrenamtliche Dienste und Krankenhäuser). Hierfür wurden die lokalen Akteure identifiziert und konzeptionell sowie organisatorisch eingebunden. Die Datenerhebung erfolgte durch geschulte Interviewerinnen und Interviewer in Form von standardisierten persönlichen Interviews im häuslichen Umfeld. Die Daten wurden in den Jahren 2015-2017 gemäß den internationalen Standards nach ICHOM bei Studieneinschluss (t0), nach 6 Monaten (t6) sowie nach 12 (t12) 24 (t24) und 36 Monaten (t36) erhoben [15].

\section{Datenschutz}

Die MmD und deren Angehörige wurden vor Studieneinschluss umfassend aufgeklärt. Schriftliche Einverständniserklärungen wurden von den Teilnehmenden oder deren gesetzlichen Vertreterinnen bzw. Vertretern eingeholt.

\section{Ethikvotum}

Vor Beginn der Studie wurde das Votum der Ethikkommission der Medizinischen Fakultät der Friedrich-Alexander-Universität Erlangen-Nürnberg eingeholt (Zeichen: 141_12B).

\section{Erhebungsinstrumente}

Es wurden soziodemografische Daten der MmD erhoben. Kognitive Fähigkeiten wurden mit dem Mini-Mental-Status-Test (MMST; Range: 0 -30) erfasst [16]. Der Schweregrad der Demenz wurde in folgende Kategorien unterteilt: > 26 = keine kognitiven Einschränkungen, 18-26 = leichte Demenz, 10-17 mittelschwere Demenz, $<10=$ schwere Demenz. Tombaugh et al. schlagen für die Kategorisierung der leichten Demenz zwar einen Bereich von 18-23 vor [17], jedoch wurde festgestellt, dass der MMST insbesondere im Bereich über 20 Punkten eine geringe Sensitivität aufweist [17-19]. Aus diesem Grund wurde die obere Grenze für die Einteilung der leichten Demenz in Anlehnung an nationale und internationale Studien und Leitlinien bei 26 Punkten gesetzt [20-22]. Alltagspraktische Fähigkeiten wurden mit dem Alzheimer's Disease Cooperative Study Group - Activities of Daily Living Inventory (ADCS-ADL; Range: 0-78, höhere Werte entsprechen stärker ausgeprägen alltagspraktischen Fähigkeiten) [23] erfasst. Die Symptombelastung wurde in Form von psychischen und Verhaltensauffälligkeiten wie z. B. Angst, Depression, abweichendes motorisches Verhalten, Aggression, Reizbarkeit, Schlafstörungen, Störungen des Appetits, Wahnvorstellungen oder Halluzinationen mit dem Neuropsychiatric Inventory (NPI; Range: 0-144, höhere Werte entsprechen stärkerer Ausprägung) [24] erhoben. Das auf körperlichen Komorbiditäten basierende Mortalitätsrisiko wurde mit dem Charlson Comorbidity Index (Charlson-Index; theoretische Range: 1-37, höhere Werte entsprechen stärkerer Ausprägung, wobei bereits ein Wert $\geq 5$ mit einem nahezu 85-prozentigem 1-Jahres-Mortalitätsrisiko assoziiert wird) [25] ermittelt. Der Charlson-Index kombiniert Anzahl und Schweregrad (bezogen auf das 1-Jahres-Mortalitätsrisiko) der Komorbiditäten. Die Inanspruchnahme des Gesundheitssystems wurde anhand des Fragebogens Resource Utilization in Dementia (RUD) [26] erfasst. Die genannten Erhebungsinstrumente sind international weit verbreitet und für den Einsatz bei Menschen mit Demenz validiert [26-34]. Gesicherte deutsche Übersetzungen liegen für alle Erhebungsinstrumente vor [26, 35-38], wenngleich spezielle Validierungsstudien der deutschsprachigen Versionen sowie Normierungen dieser international gebräuchlichen Instrumente mit Ausnahme des MMST fehlen $[38,39]$. Angehörige gaben zudem Auskünfte über Sterbeorte und Todesursachen.

\section{Statistische Analyse}

Für die Analysen erfolgte ein 1:1-Propensity Score Matching $[40,41]$ von verstorbenen und nicht-verstorbenen $\mathrm{MmD}$ über alle 3 Regionen hinweg hinsichtlich der Matching-Variablen Alter, Geschlecht, kognitiver und alltagspraktischer Fähigkeiten (MMST und ADCS-ADL) sowie der Follow-up Dauer, um vergleichbare Gruppen hinsichtlich zentraler individueller soziodemografischer Daten und der Ausprägung der Demenz nach ICD-10 [14] zu erhalten: Mithilfe des Algorithmus ,random order, nearest available pair matching method“ “ 41] wurde jeder verstorbenen Person randomisiert eine nicht verstorbene Person mit gleichem oder sehr ähnlichem Propensity Score (Schwellenwert: 0,5) zugewiesen. Der Propensity Score ist die teilnehmerspezifische Wahrscheinlichkeit für die Gruppenzugehörigkeit, geschätzt auf Basis einer logistischen Regression mit der Gruppierungsvariable als abhängige Variable und den interessierenden Matching-Variablen als unabhängige Variablen $[40,41]$. Somit fließen nur die zum Studienbeginn vorhandenen Patientenmerkmale in das Matching mit ein [40].

Da die Gruppen durch das Matching nicht mehr unabhängig voneinander sind, werden zur statistischen Analyse Verfahren für abhängige Stichproben empfohlen [40, 42]. Deshalb wurden Gruppenvergleiche bei kategorialen Variablen mittels McNemar-Tests und bei stetigen Variablen anhand von t-Tests für verbundene Stichproben berechnet. Ergänzend wurden die Effektstärkemaße Odds Ratio (OR) bzw. Cohen's d angegeben. Für jeden verstorbenen MmD wurde jeweils der letzte verfügbare Wert vor dem Tod herangezogen, um die Progression der Demenz zu berücksichtigen und eine valide Aussage über die letzte Lebensphase vor dem Tod treffen zu können. Zum Vergleich wurde von nicht verstorbenen MmD ebenfalls der, aufgrund des Matchings vergleichbare, letzte verfügbare Wert im Rahmen des Studienzeitraums von 3 Jahren verwendet. Als statistisches Signifikanzniveau wurde $5 \%$ festgelegt.

Die Daten wurden mittels SPSS Software, Version 21 (International Business Machines Corporation (IBM), Armonk, New York, USA) analysiert.

\section{Ergebnisse}

Von insgesamt 364 in BayDem befragten MmD sind 77 innerhalb des Beobachtungszeitraums von 3 Jahren verstorben. Dies entspricht einer Mortalitätsrate von 21,2\%.

Für die statistischen Analysen wurde eine Gesamtstichprobe von $\mathrm{n}=116$ in die Analyse eingeschlossen: 58 zwischen t0 (baseline) und t36 (nach 36 Monaten) verstorbene und 58 nicht verstorbene MmD, 
die mittels Propensity Score Matching $[40,41]$ parallelisiert wurden. Für 19 weitere verstorbene Personen existierte kein adäquater statistischer Zwilling. Die Baseline-Charakteristika der in die statistische Analyse eingeschlossenen MmD sind in > Tab. 1 veranschaulicht. Das gelungene Matching zeigt sich darin, dass zu Beginn keine Unterschiede zwischen verstorbenen und nicht verstorbenen MmD bestehen. In beiden Gruppen war die Demenz im Schnitt mittelschwer ausgeprägt (siehe MMST-Werte in > Tab. 1). Der Median für den Zeitraum zwischen Diagnosestellung und Tod betrug 2 Jahre und 4 Monate, zwischen Diagnosestellung und Studieneinschluss 6 Monate. Für die Anzahl der Befragungszeitpunkte betrug der Median für beide Gruppen 2 Befragungen.

MmD verstarben meist im eigenen Zuhause (36,2\%), in einem Krankenhaus (25,9\%) oder in einem Alten-/Pflegeheim (19,0\%). Niemand starb auf einer Palliativstation oder in einem Hospiz. Eine spezialisierte ambulante Palliativversorgung (SAPV) wurde im Rahmen der Versorgung am Lebensende zudem von niemandem beansprucht. Von 19,0\% lagen keine Informationen über den Sterbeort vor.

Die häufigsten Todesursachen waren Komplikationen des respiratorischen Systems (13,8\%), gefolgt von kardiovaskulären Komplikationen (12,1\%) oder einem Schlaganfall (12,1\%). Vereinzelt starben Menschen mit Demenz auch an Nierenversagen (5,2\%), Krebs (3,4\%) oder anderen Todesursachen wie multiplem Organversagen $(1,7 \%)$, inneren Blutungen $(3,4 \%)$ oder den Folgen einer Darm-OP (1,7\%). Für etwas weniger als die Hälfte der Fälle (46,5\%) lagen keine Informationen bezüglich der Todesursache vor.
Beinahe jeder zweite MmD in der letzten Lebensphase wurde innerhalb des Zeitraums von 30 Tagen vor der letzten Befragung in ein Krankenhaus eingewiesen und beinahe jeder Vierte nahm die Behandlung in einer Notaufnahme in Anspruch. Im Vergleich zu den übrigen MmD wurden sie signifikant häufiger in ein Krankenhaus eingewiesen ( 46,6 vs. $12,1 \%, p<0,001, O R=6,250$ ) oder in einer Notaufnahme eines Krankenhauses behandelt (22,4 vs. 3,4\%, $p=0,007, O R=6,500)$. Im Hinblick auf verschiedene Unterbringungsformen gab es keine signifikanten Unterschiede zwischen den Gruppen ( $>$ Tab. 2).

Die Inanspruchnahme ambulanter medizinischer und therapeutischer Leistungen von MmD in der letzten Lebensphase kann mit Ausnahme von Hausärztinnen und Hausärzten als gering betrachtet werden. Es fanden sich keine Unterschiede in der Inanspruchnahme ambulanter medizinischer und therapeutischer Leistungen zwischen den Gruppen ( $>$ Tab. 2).

In Bezug auf professionelle Unterstützungsleistungen zeigte sich, dass beinahe jeder zweite MmD in der letzten Lebensphase einen ambulanten Pflegedienst nutzte. Unterstützungsangebote wie Hauswirtschaftliche Hilfe, Essen auf Rädern, Fahrdienste für Pflege, Tagespflege, Tagesklinik sowie Betreuungsdienste wurden von $\mathrm{MmD}$ in der letzten Lebensphase mit Nutzungsraten von 1,7 bis 17,2\% vergleichsweise selten in Anspruch genommen. Deskriptiv war auffällig, dass MmD in der letzten Lebensphase knapp doppelt so häufig ambulante Pflegedienste in Anspruch nahmen als die Vergleichsgruppe, die nicht verstarb, wenngleich dieser Unterschied - berücksichtigt werden müssen dabei die relativ geringen Gruppengrößen von zwei mal 58 MmD - knapp nicht signifikant

- Tab. 1 Baseline-Charakteristika der untersuchten Menschen mit Demenz

\begin{tabular}{|c|c|c|c|c|}
\hline & $\begin{array}{l}\text { Zwischen to und } t 36 \\
\text { verstorben }(n=58)\end{array}$ & $\begin{array}{l}\text { Zwischen t0 und t36 nicht } \\
\text { verstorben }(n=58)\end{array}$ & p-Werta, b & $\begin{array}{l}\text { Gesamt } \\
(n=116)\end{array}$ \\
\hline Alter, M (SD) & $81,85(8,81)$ & $81,78(8,76)$ & 0,782 & $81,81(8,75)$ \\
\hline Frauen, n (\%) & $22(37,9)$ & $24(41,4)$ & 0,678 & $46(39,7)$ \\
\hline Kognitive Fähigkeiten (MMST), M (SD) & $14,03(8,19)$ & $14,86(6,73)$ & 0,467 & $14,45(7,47)$ \\
\hline Leichte Demenz, n (\%) & $24(41,4)$ & $24(41,4)$ & & $48(34,5)$ \\
\hline Mittelschwere Demenz, n (\%) & $11(19,0)$ & $17(29,3)$ & & $28(24,1)$ \\
\hline Schwere Demenz, n (\%) & $23(39,7)$ & $17(29,3)$ & & $40(34,5)$ \\
\hline Alltagspraktische Fähigkeiten (ADCS-ADL), M (SD) & $25,67(21,91)$ & $28,45(20,08)$ & 0,242 & $27,06(20,97)$ \\
\hline Psychische und Verhaltensauffälligkeiten (NPI), M (SD) & $31,35(23,74)$ & $25,36(24,14)$ & 0,214 & $28,40(24,01)$ \\
\hline Körperliche Komorbiditäten (Charlson-Index), M (SD) & $2,72(2,88)$ & $2,28(1,98)$ & 0,486 & $2,50(2,48)$ \\
\hline Wohnort, n (\%) & & & 0,124 & \\
\hline Stadt & $42(72,4)$ & $34(58,6)$ & & $76(65,5)$ \\
\hline Land & $16(27,6)$ & $24(41,4)$ & & $40(34,5)$ \\
\hline Höchster Schulabschluss, n (\%) & & & 0,103 & \\
\hline Abitur & $6(10,3)$ & $2(3,4)$ & & $8(6,9)$ \\
\hline Fachhochschule & $1(1,7)$ & $1(1,7)$ & & $2(1,7)$ \\
\hline Mittlere Reife & $8(13,8)$ & $11(19,0)$ & & $19(16,4)$ \\
\hline Hauptschule & $10(17,2)$ & $16(27,6)$ & & $26(22,4)$ \\
\hline Volksschule & $32(55,2)$ & $25(43,1)$ & & $57(49,1)$ \\
\hline Kein Schulabschluss & $1(1,7)$ & $3(5,2)$ & & $4(3,4)$ \\
\hline
\end{tabular}


- Tab. 2 Inanspruchnahme von Versorgungsleistungen des Gesundheitssystems zum letzten Befragungszeitpunkt

\begin{tabular}{|c|c|c|c|c|c|}
\hline & $\begin{array}{l}\text { Zwischen } t 0 \text { und } \\
t 36 \text { verstorben } \\
(n=58) n(\%)\end{array}$ & $\begin{array}{l}\text { Zwischen } \mathrm{t} 0 \text { und } \mathrm{t} 36 \\
\text { nicht verstorben } \\
(\mathrm{n}=58) \mathrm{n}(\%)\end{array}$ & p-Wert ${ }^{a, b}$ & $\begin{array}{l}\text { Odds Ratio } \\
\text { [95\%-KI] }\end{array}$ & $\begin{array}{l}\text { Gesamt } \\
(n=116) n(\%)\end{array}$ \\
\hline \multicolumn{6}{|c|}{ Stationäre medizinische Leistungen } \\
\hline Krankenhaus insgesamt & $27(46,6)$ & $7(12,1)$ & $<0,001^{* * *}$ & $\begin{array}{l}6,250[2,158 \\
24,710]\end{array}$ & $34(29,3)$ \\
\hline Notaufnahme & $13(22,4)$ & $2(3,4)$ & $0,007^{* *}$ & $\begin{array}{l}6,500[1,472 \\
59,329]\end{array}$ & $15(12,9)$ \\
\hline Geriatrie & $12(20,7)$ & $4(6,9)$ & 0,057 & $\begin{array}{l}3,667[0,969 ; \\
20,469]\end{array}$ & $16(13,8)$ \\
\hline Psychiatrie & $3(5,2)$ & $0(0,0)$ & 0,250 & $-\mathrm{c}$ & $3(2,6)$ \\
\hline Innere Medizin & $6(10,3)$ & $2(3,4)$ & 0,289 & $\begin{array}{l}3,000[0,536 \\
30,393]\end{array}$ & $8(6,9)$ \\
\hline Chirurgie & $5(10,2)$ & $0(0,0)$ & 0,063 & $-c$ & $5(4,3)$ \\
\hline Neurologie & $3(5,2)$ & $0(0,0)$ & 0,250 & $-c$ & $3(2,6)$ \\
\hline Normalstation & $4(6,9)$ & $2(3,4)$ & 0,687 & $\begin{array}{l}2,000[0,287 \\
22,110]\end{array}$ & $6(5,2)$ \\
\hline \multicolumn{6}{|c|}{$\begin{array}{l}\text { Ambulante medizinische und therapeutische } \\
\text { Leistungen }\end{array}$} \\
\hline Hausarzt/Hausärztin & $44(75,9)$ & $49(84,5)$ & 0,238 & $\begin{array}{l}0,500[0,154 ; \\
1,439]\end{array}$ & $93(80,2)$ \\
\hline Geriater/in & $4(6,9)$ & $0(0,0)$ & 0,125 & $-c$ & $4(3,4)$ \\
\hline Neurologe/Neurologin & $12(20,3)$ & $14(24,1)$ & 0,839 & $\begin{array}{l}0,846[0,343 \\
2,047]\end{array}$ & $26(22,4)$ \\
\hline Psychiater/in & $2(3,4)$ & $4(6,9)$ & 0,625 & $\begin{array}{l}0,250[0,005 \\
2,526]\end{array}$ & $6(5,2)$ \\
\hline Physiotherapeut/in & $13(22,0)$ & $12(20,7)$ & 1,000 & $\begin{array}{l}1,167[0,336 \\
4,202]\end{array}$ & $(22,9)$ \\
\hline Ergotherapeut/in & $7(12,1)$ & $4(6,9)$ & 0,549 & $\begin{array}{l}1,400[0,382 \\
5,594]\end{array}$ & $11(9,5)$ \\
\hline Sozialarbeiter/in & $0(0,0)$ & $1(1,7)$ & 1,000 & $-c$ & $1(0,9)$ \\
\hline Psychologe/Psychologin & $1(1,7)$ & $1(1,7)$ & 1,000 & $\begin{array}{l}1,000[0,013 \\
78,497]\end{array}$ & $2(1,7)$ \\
\hline \multicolumn{6}{|c|}{ Professionelle Unterstützungsangebote } \\
\hline Gemeindeschwester & $0(0,0)$ & $0(0,0)$ & - & $-c$ & $0(0,0)$ \\
\hline Ambulante Pflege & $30(51,7)$ & $19(32,8)$ & 0,082 & $\begin{array}{l}2,031[0,979 \\
4,751]\end{array}$ & $49(42,2)$ \\
\hline Hauswirtschaftliche Hilfe & $10(17,2)$ & $11(19,0)$ & 1,000 & $\begin{array}{l}1,000[0,327 \\
3,057]\end{array}$ & $21(18,1)$ \\
\hline Essen auf Rädern & $4(6,9)$ & $4(6,9)$ & 1,000 & $\begin{array}{l}1,333[0,226 \\
9,102]\end{array}$ & $8(6,9)$ \\
\hline Fahrdienste für Pflege & $7(12,1)$ & $8(13,8)$ & 1,000 & $\begin{array}{l}1,000[0,327 \\
3,057]\end{array}$ & $15(12,9)$ \\
\hline Tagespflege & $8(13,8)$ & $15(25,9)$ & 0,210 & $\begin{array}{l}0,533[0,196 \\
1,340]\end{array}$ & $23(19,8)$ \\
\hline Tagesklinik & $1(1,7)$ & $0(0,0)$ & 1,000 & $-c$ & $1(0,8)$ \\
\hline Betreuungsdienst & $4(6,9)$ & $5(8,6)$ & 1,000 & $\begin{array}{l}0,800[0,159 \\
3,717]\end{array}$ & $9(7,8)$ \\
\hline Betreuungsgruppe & $0(0,0)$ & $0(0,0)$ & - & $-^{c}$ & $0(0,0)$ \\
\hline
\end{tabular}

war (51,7 vs. 32,8\%, p=0,082, OR=2,031). Tagespflege wird zwar insgesamt seltener in Anspruch genommen, jedoch relativ häufiger von nicht verstorbenen MmD im Vergleich zu MmD in der letzten Lebensphase (13,8 vs. $25,9 \%, p=0,210, O R=0,533)$. Die übri- gen Unterstützungsleistungen sind weder deskriptiv noch inferenzstatistisch auffällig ( $>$ Tab. 2 ).

Anzahl und Schweregrad der körperlichen Komorbiditäten waren bei $\mathrm{MmD}$ in der letzten Lebensphase zum letzten Befragungszeit- 
> Tab. 3 Kognitive und alltagspraktische Fähigkeiten, körperliche Komorbiditäten und psychische und Verhaltensauffälligkeiten zum letzten Befragungszeitpunkt.

\begin{tabular}{|c|c|c|c|c|c|}
\hline & $\begin{array}{l}\text { Zwischen t0 und t36 } \\
\text { verstorben ( } n=58) M(S D)\end{array}$ & $\begin{array}{l}Z \text { wischen t0 und t } 36 \text { nicht } \\
\text { verstorben }(n=58) M(S D)\end{array}$ & p-Wert ${ }^{a, b}$ & $\begin{array}{l}\text { Cohen's d } \\
\text { [95\%-KI] }\end{array}$ & $\begin{array}{l}\text { Gesamt } \\
(n=116) M(S D)\end{array}$ \\
\hline Kognitive Fähigkeiten (MMST) & $12,34(8,17)$ & $13,38(7,06)$ & 0,360 & $\begin{array}{l}-0,136[-0,501 \\
0,228]\end{array}$ & $12,78(7,57)$ \\
\hline $\begin{array}{l}\text { Alltagspraktische Fähigkeiten } \\
\text { (ADCS-ADL) }\end{array}$ & $19,24(19,88)$ & $23,55(17,88)$ & 0,133 & $\begin{array}{l}-0,228[-0,593 \\
0,137]\end{array}$ & $21,39(18,95)$ \\
\hline $\begin{array}{l}\text { Körperliche Komorbiditäten } \\
\text { (Charlson-Index) }\end{array}$ & $2,75(2,86)$ & $1,80(1,34)$ & $0,030^{*}$ & $0,425[0,057 ; 0,793]$ & $2,25(2,27)$ \\
\hline $\begin{array}{l}\text { Psychische und Verhaltensauf- } \\
\text { fälligkeiten (NPI) }\end{array}$ & $31,67(22,32)$ & $24,78(23,13)$ & 0,118 & $\begin{array}{l}0,303[-0,063 \\
0,669]\end{array}$ & $27,70(22,00)$ \\
\hline
\end{tabular}

punkt signifikant stärker ausgeprägt als bei den übrigen $\mathrm{MmD}$ (Charlson-Index: $M=2,75$ vs. $M=1,80, p=0,030$, Cohen's $d=0,425)$. Die Matching-Variablen kognitive sowie alltagspraktische Fähigkeiten unterschieden sich auch zum letzten Befragungszeitpunkt nicht signifikant zwischen den Gruppen. Die deskriptiv stärkere Ausprägung der Symptombelastung in Form von psychischen und Verhaltensauffälligkeiten bei MmD in der letzten Lebensphase erwies sich als statistisch nicht signifikant (NPI-Wert: $M=31,67$ vs. $M=24,77, p=0,118$, Cohen's d=0,303) ( Tab. 3).

\section{Diskussion}

Bei BayDem handelte es sich um eine multizentrische Längsschnittstudie, die nicht nur Verlaufsdaten über Menschen mit Demenz erfasste, sondern auch Informationen zur Versorgungssituation ermittelte. Die Studie wurde in 3 verschiedenen bayerischen Regionen mit unterschiedlichen Bevölkerungsentwicklungen durchgeführt und berücksichtigte dabei sowohl städtische als auch ländliche Gebiete. Insbesondere die unterschiedlichen Rekrutierungsquellen und die Stichprobengröße ermöglichen es, für die Studienregion verlässliche Schlüsse zu ziehen. Auf Grundlage dieser Studienkonzeption konnten neue Erkenntnisse über die Symptombelastung, Todesumstände sowie Inanspruchnahme des Gesundheitssystems von $\mathrm{MmD}$ in der letzten Lebensphase gewonnen werden. Die Fragestellung war bisher, sowohl national wie international betrachtet, selten Gegenstand von methodisch hochwertigen Studien [3, 5, 8].

\section{Sterbeorte}

Gerade der Befund, dass MmD in der letzten Lebensphase in den meisten Fällen zuhause, in einem Krankenhaus oder in einem Altenoder Pflegeheim starben, nie jedoch auf einer spezialisierten Palliativstation oder im Rahmen einer spezialisierten ambulanten Palliativversorgung, unterstreicht die bereits in vorherigen Arbeiten beschriebene geringe Inanspruchnahme palliativer Versorgungsleistungen von MmD in der letzten Lebensphase in Deutschland [43] sowie international [44, 45]. Diese könnte zum einen darauf zurückzuführen sein, dass die Identifikation palliativer Bedarfe gerade bei Menschen mit nicht-onkologischen Erkrankungen häufig eine große Herausforderung für Ärztinnen und Ärzte darstellt, weshalb in der Vergangenheit bereits verschiedene Instrumente wie beispielsweise das Supportive and Palliative Care Indicators Tool (SPICT) für eine systematische und valide Identifikation entwickelt wurden [46]. Zum anderen fehlen Ärztinnen und Ärzten aufgrund des Mangels an evidenzbasierten Leitlinien [3, 5-8] auch fundierte Referenzen für die Beratung von Betroffenen und Angehörigen [3]. Möglicherweise vermitteln sie dadurch palliative Versorgungsangebote seltener weiter, sodass pflegende Angehörige eines MmD häufig nicht von den Vorteilen einer spezialisierten Palliativversorgung erfahren und die Nachfrage in der Folge gering bleibt.

\section{Todesursachen}

In den meisten Fällen starben MmD in der vorliegenden Studie an Komplikationen des respiratorischen Systems, kardiovaskulären Komplikationen oder aufgrund eines Schlaganfalls, was sich im Vergleich zu den übrigen MmD in einer signifikant höheren Ausprägung an körperlichen Komorbiditäten zum letzten Befragungszeitpunkt vor dem Tod widerspiegelte. Dies steht im Einklang mit der bisherigen Literatur zu unmittelbaren Todesursachen bei MmD [4, 47-49] und gibt zudem Hinweise darauf, dass MmD nicht an der fortschreitenden Demenz selbst sterben, sondern an den damit assoziierten Komplikationen. Hierfür spricht, dass sich die in der vorliegenden Arbeit untersuchten MmD in der letzten Lebensphase sowohl zu Beginn als auch zum letzten Befragungszeitpunkt nicht signifikant hinsichtlich kognitiver und alltagspraktischer Fähigkeiten, d. h. in der Ausprägung ihrer Demenz, von den nicht verstorbenen (gematchten) MmD unterschieden. Dies lässt den Schluss zu, dass die Progredienz der Demenz in beiden gematchten Gruppen vergleichbar war.

\section{Symptombelastung}

Die Symptombelastung in Form von psychischen und Verhaltensauffälligkeiten wie z. B. Angst, Depression, abweichendes motorisches Verhalten, Aggression, Reizbarkeit, Schlafstörungen, Störungen des Appetits, Wahnvorstellungen oder Halluzinationen, war sowohl zu Beginn der Studie als auch zum letzten Erhebungszeitpunkt bei MmD in der letzten Lebensphase stark ausgeprägt $[31,50]$. Im Vergleich zu den übrigen MmD war sie zum letzten Befragungszeitpunkt deskriptiv stärker ausgeprägt. Dies steht im Einklang mit der bisherigen Literatur [3, 5, 51]. 


\section{Inanspruchnahme von Versorgungsleistungen des Gesundheitssystems}

Die deutlich höhere Inanspruchnahme von Krankenhäusern oder Notaufnahmen lässt sich durch die stärkere Ausprägung körperlicher Komorbiditäten von MmD in der letzten Lebensphase erklären [52]. Dieser Sachverhalt ist auch mit unerwünschten Begleiterscheinungen verbunden, da MmD in der letzten Lebensphase in Krankenhäusern signifikant seltener an palliative Versorgungsangebote überwiesen werden als andere Patienten [53] und Krankenhausaufenthalte für MmD in der letzten Lebensphase mit starken psychischen sowie physischen Belastungen einhergehen [54]. Für den Fall, dass MmD an palliative Versorgungsangebote überwiesen werden, sind diese darüber hinaus meist qualitativ schlechter als für Menschen mit Tumorerkrankungen [45].

Trotz ausgeprägter psychischer und Verhaltensauffälligkeiten erfolgte nur selten eine Behandlung auf einer spezialisierten (psychiatrischen) Station und auch im ambulanten Bereich wurde meist lediglich die Hausärztin bzw. der Hausarzt konsultiert. Allerdings können gerade Hausärztinnen und Hausärzte durch eine Behandlungskontinuität über viele Jahre hinweg [55] oder durch das Angebot von Hausbesuchen [56] für MmD, die keiner SAPV bedürfen oder keinen Zugang zu dieser Versorgung finden, eine wichtige Säule und Konstante in der letzten Lebensphase darstellen. Gleichwohl zeigt eine Übersichtsarbeit, dass nicht-onkologische Patienten wie MmD im Rahmen einer sogenannten allgemeinen ambulanten Palliativversorgung (AAPV) durch Hausärztinnen und Hausärzte häufig keine angemessene Versorgung erhalten [57]. Dies ist ein Hinweis darauf, dass Weiterentwicklungs- sowie Verbesserungsbedarf hinsichtlich der Versorgungsqualität in diesem Bereich besteht. Professionelle Unterstützungsangebote wie Tagespflegen, Betreuungsdienste oder hauswirtschaftliche Hilfe wurden ebenfalls nur vereinzelt genutzt.

Diese Ergebnisse stehen in Übereinstimmung mit bisherigen Befunden zur Inanspruchnahme des Gesundheitssystems von MmD in der letzten Lebensphase [48] und erscheinen im Kontext des nahenden Lebensendes mit häufigen Krankenhauseinweisungen und stark ausgeprägten körperlichen Komorbiditäten nachvollziehbar. Zurückgeführt werden könnte die beschriebene geringe Inanspruchnahme ambulanter medizinischer und therapeutischer sowie professioneller Unterstützungsangebote darüber hinaus auf die Angst der Betroffenen vor Verlust der Unabhängigkeit und ihres selbstbestimmten Lebens [58]. Auf Seiten der Angehörigen stellen fehlende Kenntnisse über vorhandene Angebote eine in der Literatur häufig genannte Barriere der Inanspruchnahme dar [59]. Doch auch Stigmata sowie inadäquates Wissen über Demenz zählen zu relevanten Barrieren der Hilfesuche [60].

\section{Limitationen}

Die Rekrutierung der MmD fand in 3 Regionen Bayerns über vielfältige Einrichtungen statt, mit dem Ziel, eine breite Datenbasis zu erhalten. Nichtsdestotrotz kann durch den regionalen Fokus auf bestimmte Einrichtungen eine Selektion von Teilnehmerinnen und Teilnehmern mit spezifischen Versorgungshintergründen nicht ausgeschlossen werden. Todesursachen und Sterbeorte wurden zudem von den Angehörigen der Verstorbenen erfragt, wobei nicht von allen die betreffenden Informationen eingeholt werden konnten. Dies schränkt die Verallgemeinerbarkeit der Ergebnisse ein.
In der vorliegenden Studie wurde die Inanspruchnahme der AAPV durch Hausärztinnen und Hausärzte nicht erfragt. Da diese jedoch überwiegend von Menschen mit nicht-onkologischen Erkrankungen am Lebensende in Anspruch genommen wird [57], könnte dies zu einer Unterschätzung der tatsächlichen Inanspruchnahme der existierenden Palliativversorgung geführt haben.

Um Vergleiche mit einer Gruppe nicht verstorbener MmD durchführen zu können, wurde eine gleich große, parallelisierte Personengruppe nach der Methode des Propensity Score Matching erzeugt. Dies ist zwar eine methodisch angemessene Vorgehensweise, die Zusammensetzung der parallelisierten Gruppen ist jedoch abhängig davon, nach welchen Variablen das Matching durchgeführt wird. Dies können nur bekannte und tatsächlich gemessene Patientenmerkmale sein [40].

In zukünftigen Studien sollte darüber hinaus das Vorliegen von Patientenverfügungen oder Vorsorgevollmachten im Rahmen des sogenannten Advance Care Planning (ACP) [61] erfragt werden, da ACP als wichtige Einflussgröße bei Entscheidungsprozessen im Hinblick auf die Behandlung und den Erhalt der Autonomie der Betroffenen am Lebensende betrachtet wird [7]. Zukünftige Forschung zur Palliativversorgung von Menschen mit Demenz sollte zudem spezifische palliative Assessment-Tools wie z. B. das SPICT [46] verwenden, um relevante Palliativbedarfe dieser Zielgruppe noch weitreichender zu erfassen und damit die Versorgungsqualität der Palliativversorgung langfristig zu verbessern.

\section{FAZIT FÜR DIE PRAXIS}

- Es sollten evidenzbasierte Leitlinien für eine angemessene, den speziellen Bedürfnissen von MmD in der letzten Lebensphase entsprechende, Palliativversorgung entwickelt werden.

- Die Entwicklung evidenzbasierter Leitlinien könnte fundierte Bezugspunkte für die Beratung von Betroffenen und Angehörigen schaffen.

- Bei der Entwicklung evidenzbasierter Leitlinien sollten stark ausgeprägte psychische und Verhaltensauffälligkeiten bei MmD in der letzten Lebensphase ebenso berücksichtigt werden wie ausgeprägte körperlichen Komorbiditäten.

- Angesichts häufiger Krankenhausaufenthalte von MmD in der letzten Lebensphase sollte zudem ein Fokus auf die Entwicklung fachlicher Empfehlungen für den stationären Bereich (Akutkrankenhaus, Palliativstation) gelegt werden.

\section{Förderung}

Das Projekt BayDem wurde durch das Bayerische Staatsministerium für Gesundheit und Pflege (StMGP) gefördert (Förderkennzeichen: G42b-G8092.9-2014/10-7). 


\section{Danksagung}

Besonderer Dank gilt allen Teilnehmerinnen und Teilnehmern, insbesondere den Menschen mit Demenz und deren pflegenden Angehörigen und Betreuerinnen und Betreuern. Sie haben Zeit und Mühe auf sich genommen und durch ihre engagierte Teilnahme zum Erfolg des Bayerischen Demenz Survey erheblich beigetragen. Die Autorinnen und Autoren danken außerdem allen beteiligten Kooperationspartnerinnen und Kooperationspartnern für die engagierte Mitarbeit im Bayerischen Demenz Survey. Die vorliegende Arbeit wurde von André Kratzer als Teil der Anforderungen zur Erlangung des akademischen Grades „Dr. rer. biol. hum. “ an der Medizinischen Fakultät der Friedrich-Alexander-Universität ErlangenNürnberg (FAU) angefertigt.

\section{Interessenkonflikt}

Die Autorinnen/Autoren geben an, dass kein Interessenkonflikt besteht.

\section{Literatur}

[1] Prince M, Wimo A, Guerchet M et al. World Alzheimer Report 2015. The global impact of dementia (2015). Im Internet: https://www.alz. co.uk/research/WorldAlzheimerReport2015.pdf; Zugriff: 02.01.2019

[2] Bickel H. Informationsblatt 1. Die Häufigkeit von Demenzerkrankungen 2018; Im Internet: https://www.deutsche-alzheimer.de/fileadmin/ alz/pdf/factsheets/infoblatt1_haeufigkeit_demenzerkrankungen_ dalzg.pdf; Zugriff: 29.10.2018

[3] Diehl-Schmid J, Riedl L, Rüsing U et al. Palliativversorgung von Menschen mit fortgeschrittener Demenz. Nervenarzt 2018; 89: 524-529

[4] Koopmans RT, van der Sterren KJ, van der Steen JT. The 'natural' endpoint of dementia: death from cachexia or dehydration following palliative care? Int J Geriatr Psychiatry 2007; 22: 350-355

[5] Pinzon LCE, Claus M, Perrar KM et al. Todesumstände von Patienten mit Demenz-Symptombelastung, Betreuungsqualität und Sterbeort. Dtsch Arztebl 2013; 110: 195-202

[6] van der Steen JT, Radbruch L, Hertogh CM et al. White paper defining optimal palliative care in older people with dementia: a Delphi study and recommendations from the European Association for Palliative Care. Palliat Med 2014; 28: 197-209

[7] Zieschang T, Oster P, Pfisterer $\mathrm{M}$ et al. Palliativversorgung von Menschen mit Demenz. Z Gerontol Geriat 2012; 45: 50-54

[8] van der Steen JT. Dying with dementia: what we know after more than a decade of research. J Alzheimers Dis 2010; 22: 37-55

[9] Sepulveda C, Marlin A, Yoshida T et al. Palliative Care: the World Health Organization's global perspective. J Pain Symptom Manage 2002; 24: 91-96

[10] Diop MS, Rudolph JL, Zimmerman KM et al. Palliative care interventions for patients with heart failure: a systematic review and meta-analysis. J Palliat Med 2017; 20: 84-92

[11] Lorenz KA, Lynn J, Dy SM et al. Evidence for improving palliative care at the end of life: a systematic review. Ann Intern Med 2008; 148: 147-159

[12] Murphy E, Froggatt K, Connolly $S$ et al. Palliative care interventions in advanced dementia. Cochrane Database Sys Rev 2016; 12: Cd011513
[13] Schaller S, Marinova-Schmidt V, Setzer M et al. Bayerischer Demenz Survey: Methodik einer Längsschnitterhebung zur Versorgungssituation bei Demenz. Gesundheitswesen 2015; 77: 712

[14] Dilling H, Mombour W, Schmidt MH., Hrsg. Internationale Klassifikation psychischer Störungen. ICD-10 Kapitel V (F). Klinisch-diagnostische Leitlinien. 8. Aufl. Bern: Huber; 2011

[15] International Consortium for Health Outcomes Measurement (ICHOM). Dementia. Data collection reference guide. Version 1.0.1. Im Internet: https://ichom.org/files/medical-conditions/dementia/ dementia-reference-guide.pdf; Zugriff: 31.10.2018

[16] Folstein MF, Folstein SE, McHugh PR. "Mini-mental state". A practical method for grading the cognitive state of patients for the clinician. J Psychiatr Res 1975; 12: 189-198

[17] Tombaugh TN, McIntyre NJ. The mini-mental state examination: a comprehensive review. J Am Geriatr Soc 1992; 40: 922-935

[18] Ihl R, Frolich L, Dierks T et al. Differential validity of psychometric tests in dementia of the Alzheimer type. Psychiatry research 1992; 44: 93-106

[19] Nasreddine ZS, Phillips NA, Bedirian V et al. The Montreal Cognitive Assessment, MoCA: a brief screening tool for mild cognitive impairment. J Am Geriatr Soc 2005; 53: 695-699

[20] Hager K, Henneges C, Schneider E et al. Alzheimer-Demenz: Verlauf und Belastung der Pflegepersonen : 18-Monats-Daten der deutschen Teilnehmer der GERAS-Studie. Nervenarzt 2018; 89: 431-442

[21] National Institute for Health and Care Excellence. Donepezil, galantamine, rivastigmine and memantine for the treatment of Alzheimer's disease (2011). Im Internet: https://www.nice.org.uk/ guidance/ta217/chapter/2-clinical-need-and-practice; Zugriff: 08. Januar 2019

[22] Deutsche Gesellschaft für Psychiatrie und Psychotherapie Psychosomatik und Nervenheilkunde (DGPPN), Deutsche Gesellschaft für Neurologie (DGN). S3-Leitlinie "Demenzen": Langversion (2016). Im Internet: https://www.dgppn.de/_Resources/Persistent/ade50e44afc7eb8024e7f65ed3f44e995583c3a0/S3-LL-Demenzen-240116. pdf; Zugriff: 22. Juni 2018

[23] Galasko D, Bennett D, Sano M et al. An inventory to assess activities of daily living for clinical trials in Alzheimer's disease. The Alzheimer's Disease Cooperative Study. Alzheimer Dis Assoc Disord 1997; 11 Suppl 2: 33-39

[24] Cummings JL, Mega M, Gray K et al. The Neuropsychiatric Inventory: comprehensive assessment of psychopathology in dementia. Neurology 1994; 44: 2308-2314

[25] Charlson ME, Pompei P, Ales KL et al. A new method of classifying prognostic comorbidity in longitudinal studies: development and validation. J Chronic Dis 1987; 40: 373-383

[26] Wimo A, Gustavsson A, Jonsson L et al. Application of Resource Utilization in Dementia (RUD) instrument in a global setting. Alzheimers Dement 2013; 9: 429-435

[27] Creavin ST, Wisniewski S, Noel-Storr AH et al. Mini-Mental State Examination (MMSE) for the detection of dementia in clinically unevaluated people aged 65 and over in community and primary care populations. Cochrane Database Sys Rev 2016, doi:10.1002/14651858.CD011145.pub2 Cd011145

[28] Tsoi KK, Chan JY, Hirai HW et al. Cognitive tests to detect dementia: a systematic review and meta-analysis. JAMA Intern Med 2015; 175: $1450-1458$

[29] Desai AK, Grossberg GT, Sheth DN. Activities of daily living in patients with dementia: clinical relevance, methods of assessment and effects of treatment. CNS Drugs 2004; 18: 853-875

[30] Bentvelzen A, Aerts L, Seeher K et al. A comprehensive review of the quality and feasibility of dementia assessment measures: the Dementia Outcomes Measurement Suite. J Am Med Dir Assoc 2017; 18: 826-837 
[31] Kaufer DI, Cummings JL, Ketchel P et al. Validation of the NPI-Q, a brief clinical form of the Neuropsychiatric Inventory. J Neuropsychiatry Clin Neurosci 2000; 12: 233-239

[32] Frenkel W], Jongerius EJ, Mandjes-van Uitert M] et al. Validation of the Charlson Comorbidity Index in acutely hospitalized elderly adults: a prospective cohort study. J Am Geriatr Soc 2014; 62: 342-346

[33] Chen T-B, Yiao S-Y, Sun Y et al. Comorbidity and dementia: a nationwide survey in Taiwan. PLoS One 2017; 12: e0175475

[34] de Groot V, Beckerman H, Lankhorst G] et al. How to measure comorbidity. a critical review of available methods. J Clin Epidemiol 2003; 56: 221-229

[35] Leibniz-Zentrum für Psychologische Information und Dokumentation (ZPID). ADCS-ADL - ADCS-Inventar zu Aktivitäten des täglichen Lebens (PSYNDEX Tests Info) (2018). Im Internet: https://www.psyndex.de/ retrieval/PSYNDEXTests.php?id=9007062; Zugriff: 01.04.2019

[36] Leibniz-Zentrum für Psychologische Information und Dokumentation (ZPID). NPI - Neuropsychiatrisches Inventar (PSYNDEX Tests Review). (2018). Im Internet: https://www.zpid.de/retrieval/PSYNDEXTests. php?id = 9004911; Zugriff: 01.04.2019

[37] Online P. Charlson-Komorbiditätsindex (CCI) (2018). Im Internet: https://www.pschyrembel.de/Charlson-Komorbidit\%C3\%A4tsindex/ KOPUP; Zugriff: 22.07.2019

[38] Kessler J, Markowitsch HJ, Denzler P. MMST - Mini-Mental-Status-Test. Deutschsprachige Fassung. Weinheim: Beltz Test; 1990

[39] Albrecht D, Pendergrass A, Becker C et al. Interventionsstudien zur Unterstützung häuslicher Pflege. Empfehlungen zu Endpunkten und Assessment-Instrumenten bei kognitiv eingeschränkten Pflegebedürftigen. Z Gerontol Geriat 2018; 51: 642-649

[40] Kuss O, Blettner M, Börgermann J. Propensity Score-eine alternative Methode zur Analyse von Therapieeffekten. Dtsch Arztebl 2016; 113: 35-36

[41] Bacher J. Statistisches Matching: Anwendungsmöglichkeiten, Verfahren und ihre praktische Umsetzung in SPSS. 2002; Im Internet: https://www.gesis.org/fileadmin/upload/forschung/publikationen/ zeitschriften/za_information/ZA-Info-51.pdf; Zugriff: 05.11.2018

[42] Austin PC. Comparing paired vs non-paired statistical methods of analyses when making inferences about absolute risk reductions in propensity-score matched samples. Stat Med 2011; 30: 1292-1301

[43] Genz H, Jenetzky E, Hauer K et al. Palliative Geriatrie. Z Gerontol Geriat 2010; 43: 369-375

[44] Lo AT, Karuza J, Berall A et al. Prevalence of Dementia in a Geriatric Palliative Care Unit. Am J Hosp. Palliat Med 2018; 35: 799-803

[45] Martinsson L, Lundström S, Sundelöf J. Quality of end-of-life care in patients with dementia compared to patients with cancer: A population-based register study. PLoS One 2018; 13: e0201051

[46] Highet G, Crawford D, Murray SA et al. Development and evaluation of the Supportive and Palliative Care Indicators Tool (SPICT): a mixedmethods study. BMJ Support Palliat Care 2014; 4: 285-290
[47] Brunnström HR, Englund EM. Cause of death in patients with dementia disorders. Eur J Neurol 2009; 16: 488-492

[48] Sampson EL, Candy B, Davis S et al. Living and dying with advanced dementia: a prospective cohort study of symptoms, service use and care at the end of life. Palliat Med 2018; 32: 668-681

[49] Park J. Mortality from Alzheimer's disease in Canada: A multiple-causeof-death analysis, 2004 to 2011. Health Rep 2016; 27: 17-21

[50] Mega MS, Cummings JL, Fiorello T et al. The spectrum of behavioral changes in Alzheimer's disease. Neurology 1996; 46: 130-135

[51] Gill TM, Gahbauer EA, Han L et al. Trajectories of disability in the last year of life. New Engl J Med 2010; 362: 1173-1180

[52] Prince M, Comas-Herrera A, Knapp M et al. World Alzheimer Report 2016. Improving healthcare for people living with dementia. Coverage, Quality and costs now and in the future. 2016; Im Internet: https:// www.alz.co.uk/research/WorldAlzheimerReport2016.pdf; Zugriff: 14.06.2018

[53] Fogg C, Griffiths P, Meredith P et al. Hospital outcomes of older people with cognitive impairment: An integrative review. Int J Geriatr Psychiatry 2018, doi:10.1002/gps.4919

[54] Aminoff BZ, Adunsky A. Dying dementia patients: too much suffering, too little palliation. Am J Hosp Palliat Med 2005; 22: 344-348

[55] Pereira Gray DJ, Sidaway-Lee K, White E et al. Continuity of care with doctors-a matter of life and death? A systematic review of continuity of care and mortality. BMJ Open 2018; 8: e021161

[56] Snijder E, Kersting M, Theile G et al. Hausbesuche: Versorgungsforschung mit hausärztlichen Routinedaten von 158000 Patienten. Gesundheitswesen 2007; 69: 679-685

[57] Afshar K, Geiger K, Müller-Mundt G et al. Hausärztliche Palliativversorgung bei nichtonkologischen Patienten. Schmerz 2015; 29: 604-615

[58] Stephan A, Bieber A, Hopper L et al. Barriers and facilitators to the access to and use of formal dementia care: findings of a focus group study with people with dementia, informal carers and health and social care professionals in eight European countries. BMC Geriatr 2018; 18: 131

[59] Phillipson L, Jones SC, Magee C. A review of the factors associated with the non-use of respite services by carers of people with dementia: implications for policy and practice. Health Soc Care Community 2014; 22: 1-12

[60] Werner P, Goldstein D, Karpas DS et al. Help-seeking for dementia: a systematic review of the literature. Alzheimer Dis Assoc Disord 2014; 28: $299-310$

[61] Dening KH, Jones L, Sampson EL. Advance care planning for people with dementia: a review. Int Psychogeriatr 2011; 23: 1535-1551 